\title{
The Necessity of Using Heparin in the UltraTag RBC Kit When Tagging Blood for a Nuclear Medicine Study
}

\author{
Sarah R. Pigmon ${ }^{1}$, Kara D. Weatherman ${ }^{2}$, Nicholas C. Brehl ${ }^{1}$, and Cybil J. Nielsen ${ }^{1}$ \\ ${ }^{1}$ Indiana University School of Medicine, Indianapolis, Indiana; and ${ }^{2}$ Purdue University College of Pharmacy, West Lafayette, Indiana
}

The purpose of this research was to evaluate the need to use heparin when preparing an UltraTag red blood cell (RBC) kit for a nuclear medicine study. Methods: Nonheparinized blood samples $(n=15)$ and heparinized blood samples $(n=15)$ were added to UltraTag RBC kits. The samples were examined for macroscopic blood clotting and microscopic platelet clumping. As a control, samples with heparin $(n=15)$ and without heparin ( $n=15$ ) were used to help evaluate the effectiveness of the anticoagulant properties within the UltraTag RBC kit (sodium citrate) and whether those properties played a role in preventing clots or clumps. To detect clotting, the wooden applicator stick method was used. To detect clumping, blood smears were evaluated using a light microscope. The two samples were compared for presence of clots and clumps. Fisher exact testing was used to evaluate the significance of the data. Results: For the UltraTag RBC group, 2 of the 15 nonheparinized samples clotted and none of the 15 heparinized samples clotted; for the control group, 2 of the 15 nonheparinized samples clotted and none of the 15 heparinized samples clotted. For the Ultra-Tag RBC group, 3 of the 15 nonheparinized samples clumped and 3 of the 15 heparinized samples clumped; for the control group, 15 of the 15 nonheparinized samples clumped and 10 of the 15 heparinized samples clumped. Conclusion: When heparin is not used, the Ultra-Tag RBC kit is more likely to form clots. Heparin should always be used when preparing an Ultra-Tag RBC kit for a nuclear medicine study.

Key Words: UltraTag; macroscopic clots; microscopic platelet clumping; heparin

J Nucl Med Technol 2016; 44:230-233

DOI: 10.2967/jnmt.116.177535

I nuclear medicine, some studies require blood to be withdrawn from the patient, radiolabeled via an UltraTag red blood cell (RBC) kit (Mallinckrodt Pharmaceuticals), and then injected back into the patient for imaging. Per the package insert instructions ( 1 ), an anticoagulant such as heparin or acid citrate dextrose is to be added to the syringe before blood is withdrawn from the patient to prevent blood clots from forming during the

\footnotetext{
Received May 4, 2016; revision accepted Aug. 7, 2016.

For correspondence or reprints contact: Sarah R. Pigmon, Department of Radiologic and Imaging Sciences, Indiana University School of Medicine, 541

Clinical Dr., Indianapolis, IN 46202-5111.

E-mail: pigmons@umail.iu.edu

Published online Sep. 15, 2016.

COPYRIGHT (c) 2016 by the Society of Nuclear Medicine and Molecular Imaging, Inc.
}

radiolabeling process. However, in some institutions, technologists prepare the radiolabeling kit without use of anticoagulants. The rationale for this deviation from the package insert instruction includes enhanced documentation and other institution-specific issues required to acquire and use anticoagulants, recurrent heparin drug shortages, the anticoagulant components of the UltraTag RBC kit, and the fairly rapid readministration of the radiolabeled product. The current research was done to evaluate whether an anticoagulant such as heparin needs to be used to prevent the formation of blood clots during the labeling process.

The recommended amount of heparin is 10-15 units per milliliter of blood (1). In RBC tagging, heparin is an anticoagulant that reduces the chances of blood clotting (2). The body also has its own fibrinolytic system responsible for lysing blood clots. This fibrinolytic system was discovered at the beginning of the 20th century by Niewiarowski, who showed that the end products of fibrinolysis inhibit the process of coagulation (3). The UltraTag RBC kit contains sodium citrate, another common anticoagulant used in vitro (4). Despite the presence of sodium citrate in the kit and the body's own fibrinolytic system, the package insert for the kit states that the syringe used to draw blood for radiolabeling must contain heparin.

Nuclear medicine studies that commonly use the UltraTag RBC kit are multigated acquisition studies and gastrointestinalbleed studies, with the former being indicated for evaluating left ventricular function at baseline before chemotherapy (5) and the latter for detecting and localizing an acute gastrointestinal bleed (5). Although prevention of blood clots is important for the health of the patient, it is also important for the accuracy of the study. If radiolabeled blood containing a clot were to be injected into the patient, the clot could potentially block a vein, thus preventing the rest of the radiolabeled blood from getting to the appropriate area.

In this study, we evaluated macroscopic blood clotting and microscopic platelet clumping. Clots are visible to the naked eye. They appear as large mucuslike threads that are formed by the aggregation and accumulation of platelets and the formation of fibrin from fibrinogen. One of the most common side effects from a clot is acute pulmonary embolism, which accounts for 50,000 deaths annually (6). Clumps can be seen only with a microscope. The term clump is used instead of clot because only aggregated platelets are microscopically visualized. The clumps have no associated macroscopic sign of mucous threads or fibrin formation. Both clotting and 


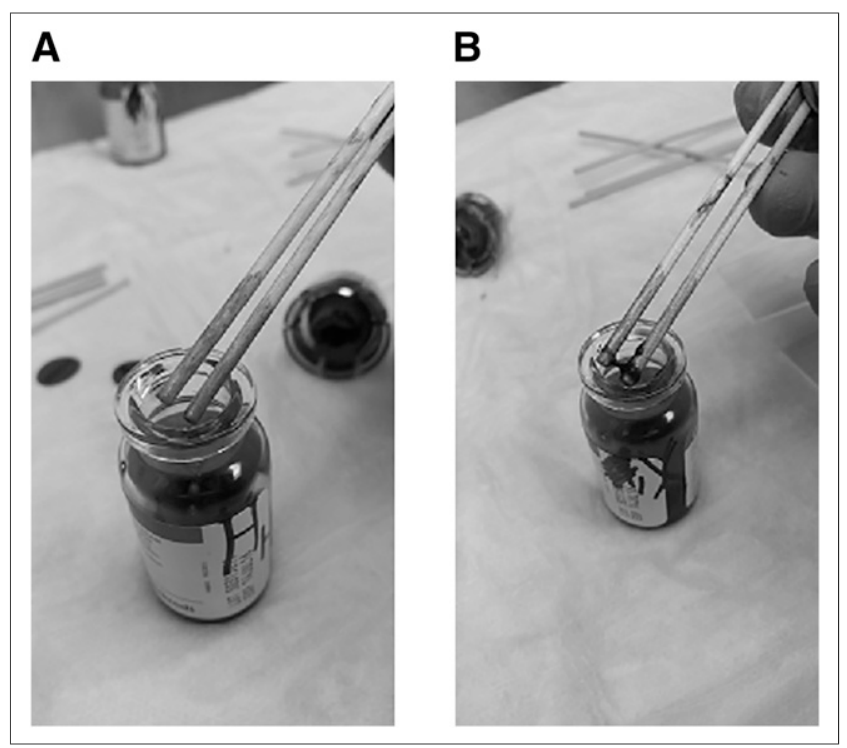

FIGURE 1. Examples of absence (A) and presence (B) of clotting.

clumping could potentially lead to pulmonary embolism, stroke, and deep vein thrombosis (7).

The purpose of this research was to evaluate the need to use heparin when preparing an UltraTag RBC kit.

\section{MATERIALS AND METHODS}

\section{Subjects}

This study was approved by the institutional review board and followed the guidelines of the Health Insurance Portability and Accountability Act. A flyer asking for volunteers was sent to the undergraduate radiologic and imaging science students at Indiana University. Fifteen volunteers were selected on a first-come, first-served basis with regard to how quickly they responded to the flyer. There were no exclusion criteria. Twelve of the volunteers were students, 2 were nuclear medicine technologists, and 1 was a nuclear pharmacist. Three were male and 12 female. Nine fell within the age range of 18-25y, and 6 fell within the age range of $26-65 \mathrm{y}$. All gave written informed consent to participate in the study.

\section{Supplies}

The supplies for each volunteer included 2 UltraTag RBC kits, 40 units of unfractionated heparin solution, $74 \mathrm{MBq}$ of ${ }^{99 \mathrm{~m}} \mathrm{Tc}$-sodium pertechnetate, 8 microscope slides, 8 wooden applicator sticks, 1 intravenous starter kit with a 20-gauge needle, 2 SmartSite vented vial access devices (BD), 2 Falcon round-bottomed tubes with lids (BD), eleven 3-mL syringes with an attaching 20-gauge needle, a dose calibrator, a lead shield, a Geiger-Müller survey meter, an Eclipse Ni-U microscope (Nikon), a Hema-Tek I 1000 WrightGiemsa pack (Siemens Healthcare Diagnostics), and pliers.

\section{Procedures}

A 20-gauge intravenous line was placed in each volunteer, and 4 samples of blood were drawn. The first was the UltraTag RBC heparinized sample ( $3 \mathrm{~mL}$ of blood drawn into a syringe containing 30 units $[0.3 \mathrm{~mL}]$ of heparin), the second was the UltraTag RBC nonheparinized sample ( $3 \mathrm{~mL}$ of blood drawn into an empty syringe), the third was the control heparinized sample $(1 \mathrm{~mL}$ of blood drawn into a syringe containing 10 units [0.1 mL] of heparin), and the fourth was the control nonheparinized sample $(1 \mathrm{~mL}$ of blood drawn into an empty syringe). The heparin had a concentration of 100 units $/ \mathrm{mL}$. Ten units of heparin per milliliter of blood were used.

The control samples were taken to determine whether the sodium citrate within the UltraTag RBC kit imparted sufficient anticoagulant properties to prevent clots or clumps. The crimp tops were removed from the commercially available UltraTag vials for ease of manipulation (herein referred to as "tubes"). The samples from the two UltraTag RBC syringes were added to separate tubes (one for the heparinized sample and one for the nonheparinized sample). The samples from the two control syringes were added to separate tubes (one for the heparinized sample and one for the nonheparinized sample). The UltraTag RBC samples were radiolabeled following the package insert guidelines, using approximately $37 \mathrm{MBq}$ of ${ }^{99 \mathrm{~m}} \mathrm{Tc}$-sodium pertechnetate.

All 4 samples of blood were evaluated for clotting and clumping. To evaluate for clotting, the wooden applicator stick method was used. Two sticks were held together like chop sticks and swirled in the tube. The sticks were then gently pulled up along the side of the tube, and once the ends of the sticks were visible, they were viewed for clotting. If clotting was seen, a "yes" was recorded on the data sheet; otherwise, a "no" was recorded. Figure 1 shows an example of clotting and an example of no clotting.

To evaluate for clumping, a blood smear was prepared. A drop of blood was placed on a microscope slide and then another slide was placed on top of the drop and the slides were pulled across each other, making a thin smear. For each of the 15 volunteers, 8 slides were prepared for the blood-smearing method: 2 for each of the 4 samples (UltraTag RBC heparinized, control heparinized, UltraTag RBC nonheparinized, and control nonheparinized). The slides were stained with a Wright-Giemsa pack using a Hema-Tek I 1000, and 20 fields of view at $\times 500$ oil magnification were analyzed by a medical laboratory scientist using an Eclipse Ni-U microscope. The fields of view were chosen at random to provide a fair representation of the slide. If the slide showed an area of clumping, a "yes" was recorded on the data sheet; otherwise, a "no" was recorded. Figure 2 shows an example of a slide negative for clumping and a slide positive for clumping.

\section{Statistical Tests}

To evaluate the data, the Fisher exact test was used. This test is useful with contingency tables of a small sample size (8). A $P$ value of less than 0.05 represented statistical significance and allowed rejection of the null hypothesis that the prevalence of clotting or clumping is not affected by whether heparin is used in the UltraTag RBC kit. The alternate hypothesis was that the use of heparin does affect the prevalence of clotting or clumping.

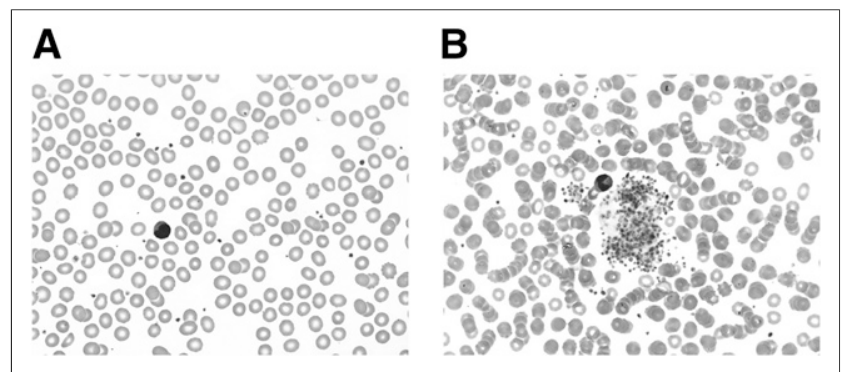

FIGURE 2. Examples of absence (A) and presence (B) of clumping. 
TABLE 1

Comparison of Positive Results With and Without Heparin

\begin{tabular}{|c|c|c|c|c|}
\hline Group & Result & With heparin & Without heparin & $P$ \\
\hline \multirow[t]{2}{*}{ UltraTag RBC } & Clotting & $0 / 15(0 \%)$ & $2 / 15(13 \%)$ & 0.48 \\
\hline & Clumping & $3 / 15(20 \%)$ & $3 / 15(20 \%)$ & 1.0 \\
\hline \multirow[t]{2}{*}{ Control } & Clotting & $0 / 15(0 \%)$ & $2 / 15(13 \%)$ & 0.48 \\
\hline & Clumping & $10 / 15(67 \%)$ & 15/15 (100\%) & 0.04 \\
\hline
\end{tabular}

\section{RESULTS}

None of the heparinized UltraTag RBC samples had clotting, and 3 had clumping. Two of the nonheparinized UltraTag RBC samples had clotting, and 3 had clumping. None of the heparinized control samples had clotting, and 10 had clumping. Two of the nonheparinized control samples had clotting, and 10 had clumping.

For comparisons of the heparinized and nonheparinized samples in the UltraTag RBC group, the $P$ value was 0.48 macroscopically and 1.0 microscopically. For comparisons of the heparinized and nonheparinized samples in the control group, the $P$ value was 0.98 macroscopically. In neither of these cases was there enough evidence to reject the null hypothesis. There was no significant difference between the heparinized and nonheparinized UltraTag RBC samples macroscopically or microscopically or between the control heparinized and nonheparinized samples macroscopically.

For the control group, there was a statistically significant difference in clumping between the heparinized and nonheparinized samples $(P=0.04)$. The null hypothesis was rejected in favor of the alternate hypothesis. The results of the Fisher exact test are given in Table 1.

The raw data are presented in Table 2. It was incidentally noted that 3 volunteers (volunteers 4, 9, and 13) had clumping in the heparinized UltraTag RBC sample that was not seen in the nonheparinized sample.

\section{DISCUSSION}

Although clumps must always be present if clots are seen, the reverse is not true: there can be clumps but no clots. Therefore, when clots were seen in this study, a "yes" was automatically recorded for clumps. When the control and UltraTag RBC groups were compared, it appeared that the anticoagulant properties within the UltraTag RBC kit (sodium citrate) were effective at preventing clumping but not effective enough to prevent clotting. In two volunteers (volunteers 1 and 14, Table 2), clots formed in the absence of heparin but not in the presence of heparin. Although this finding was not statistically significant, the addition of heparin helps minimize clot formation. There was a statistically significant difference in clumping between the control group and the UltraTag RBC group. A larger sample size could achieve statistically significant results regarding the other samples.

The fact that there were 3 volunteers (volunteers 4, 9, and 13) for whom clumping was identified with heparin but not without heparin may be due to two factors: the small volume of each sample (i.e., clumping in the nonheparinized sample may not have been identified because of its small volume) and the small amount of heparin used (because of the small volume of blood). If a larger amount of heparin had been used, this discrepancy might not have appeared.

Although our results did not reach statistical significance, they are still clinically significant. Ultimately, injecting a clot could harm the patient and affect the accuracy of the study. Further research should be done in which more samples of blood are taken from the UltraTag RBC kit to test for clumping.

One limitation of this study was the time frame between drawing the blood and exposing it to sodium citrate in the UltraTag RBC kit. Clotting and clumping are timedependent. This is a moot point with the heparinized samples because the heparin was already in the syringe when the

TABLE 2

Clotting and Clumping Results by Volunteer

\begin{tabular}{|c|c|c|c|c|}
\hline \multirow[b]{2}{*}{ Volunteer no. } & \multicolumn{2}{|c|}{ UltraTag RBC } & \multicolumn{2}{|c|}{ Control } \\
\hline & With heparin & Without heparin & With heparin & Without heparin \\
\hline 1 & - & Clotting and clumping & Clumping & Clumping \\
\hline 2 & - & - & - & Clumping \\
\hline 3 & - & - & - & Clumping \\
\hline 4 & Clumping & - & - & Clumping \\
\hline 5 & - & - & - & Clotting and clumping \\
\hline 6 & - & - & Clumping & Clumping \\
\hline 7 & - & - & Clumping & Clumping \\
\hline 8 & - & - & Clumping & Clumping \\
\hline 9 & Clumping & - & - & Clumping \\
\hline 10 & - & - & Clumping & Clumping \\
\hline 11 & - & Clumping & Clumping & Clumping \\
\hline 12 & - & - & Clumping & Clotting and clumping \\
\hline 13 & Clumping & - & Clumping & Clumping \\
\hline 14 & - & Clotting and clumping & Clumping & Clumping \\
\hline 15 & - & - & Clumping & Clumping \\
\hline
\end{tabular}


blood was drawn. Additional limitations include the small sample size and the fact that no criteria were imposed for the age range and sex of the volunteers. Research in which these limitations are controlled could be useful.

\section{CONCLUSION}

When an anticoagulant such as heparin is not used during the blood collection process, the Ultra-Tag RBC kit can produce clots despite the anticoagulant properties of some components of the kit. Heparin should always be used when preparing an Ultra-Tag RBC kit for a nuclear medicine study.

\section{DISCLOSURE}

Mallinckrodt Pharmaceuticals donated 30 UltraTag RBC kits. Radiopharmacy of Indianapolis donated $1,100 \mathrm{MBq}$ of ${ }^{99 m}$ Tc-sodium pertechnetate. Indiana University School of Medicine, Department of Radiology and Imaging Sciences, donated syringes, needles, IV starter kits, and gloves. Indiana University School of Medicine, Department of Pathology and Laboratory Medicine, donated wooden sticks and allowed use of a Nikon Eclipse Ni-U microscope, a HemaTek I 1000, and Wright-Giemsa stain. Purdue University College of Pharmacy donated heparin and microscope slides. Riley Hospital for Children at Indiana University Health allowed use of a dose calibrator, lead shield, Geiger Mueller survey meter, and hot lab. No other potential conflict of interest relevant to this article was reported.

\section{ACKNOWLEDGMENTS}

We thank Dennis J. Ernst, MT(ASCP), NCPT(NCCT), and the Center for Phlebotomy Education for education and guidance on blood clots.

\section{REFERENCES}

1. Ultratag ${ }^{\text {TM }}$ RBC kit for the preparation of technetium Tc $99 \mathrm{~m}$-labeled red blood cells [package insert]. Maryland Heights, MO: Mallinckrodt Inc.; 2015.

2. Cosmi B, Palareti G. Old and new heparins. Thromb Res. 2012;129:388-391.

3. Niewiarowski S, Prou-Wartelle O. Role du facteur contact (Facteur Hageman) dans la fibrinolyse. Thromb Diath Haemorrh. 1959;3:593.

4. Ramsay DM, Robertson EP, MacArthur E. Comparison of anticoagulants for the preservation of prothrombin time specimens. J Clin Pathol. 1977;30:766-769.

5. Farrell MB, Mantel ES, Basso DA, Thomas KS, Kerr BR. Quick-Reference Protocol Manual for Nuclear Medicine Technologists. Reston, VA: Society of Nuclear Medicine and Molecular Imaging; 2014.

6. Yoo HH, Queluz TH, El Dib R. Anticoagulant treatment for subsegmental pulmonary embolism. Cochrane Database Syst Rev. 2014;4:CD010222.

7. Fritsma G. Thrombotic disorders and laboratory assessment. In Keohane E, Smith L, Walenga J, eds. Rodak's Hematology: Clinical Principles and Applications. 5th ed. St. Louis, MO: Elsevier; 2016.

8. Petrie A, Sabin C. Medical Statistics at a Glance. 3rd ed. Hoboken, NJ: Blackwell Publishing; 2009:69. 